\title{
Die Rolle des Gehirns bei Adipositas
}

\author{
Annette Horstmann und Arno Villringer
}

\section{Zusammenfassung}

Die häufigste Ursache für Adipositas ist eine positive Energiebilanz, d.h. es wird mehr Energie über die Nahrung aufgenommen als z.B. durch körperliche Aktivität verbraucht wird. Die Ursache für steigende Adipositasraten in den Industriekulturen kann jedoch nicht ausschließlich in der veränderten Umwelt liegen, da es starke individuelle Unterschiede bezüglich der Gewichtsentwicklung gibt. Die Ursache liegt also wahrscheinlich in der Interaktion von individuellen Verhaltensmustern und der Umgebung. In diesem Kontext wird es spannend, die Rolle des Gehirns bei der Entstehung und Aufrechterhaltung der Adipositas zu untersuchen. Diese Frage rückt in letzter Zeit immer mehr in den Fokus der neurowissenschaftlichen Forschung. Der vorliegende Reviewartikel soll einen Überblick über den aktuellen Kenntnisstand im Bereich der bildgebenden Neurowissenschaften zur Adipositas beim Menschen geben. Darüber hinaus wird das Zusammenspiel von Genvarianten, Essverhalten und Gehirn im Hinblick auf Adipositas beleuchtet. Abschließend werden offene Fragen des Forschungsfeldes zusammengefasst, in deren Beantwortung das Forschungsfeld in naher Zukunft investieren sollte.

\begin{abstract}
,The most common cause for obesity is a positive energy balance, i.e. more energy is being consumed than is expended. The rise in obesity rates cannot be explained on the basis of our obesogenic environment alone, because large interindividual differences in weight status exist between people. Therefore, the cause is most probably to be found in an interaction between individual behaviour and our changed environment. This warrants the investigation of the brain's role in the development and maintenance of obesity that indeed has become a growing field in the neurosciences. This article will give an overview about the findings in neuroimaging associated with human obesity. Further, this article will elucidate the relationship between common genetic variation, eating behaviour and brain structure in the context of obesity. Finally, important open questions in the field will be summarised.
\end{abstract}

Keywords: obesity; brain structure; functional connectivity; SNPs; reinforcement

\section{Einführung}

Adipositas bezeichnet starkes Übergewicht, welches durch eine über das normale Maß hinausgehende Vermehrung des Körperfettes gekennzeichnet ist. Nach der Definition der World Health Organization (WHO) gilt ein Mensch ab einem Körpermasseindex (Body Mass Index, BMI) von über $30 \mathrm{~kg} / \mathrm{m}^{2}$ als adipös. Adipositas ist ein hoher Risikofaktor für die Entwicklung von z.B. Herz-Kreislauferkrankungen, Bluthochdruck, Diabetes mellitus Typ 2 und erhöht darüber hinaus das Risiko, im Laufe des Lebens an verschiedenen Arten von Krebs oder Demenz zu erkranken. Laut einer repräsentativen Studie aus den Jahren 2008-11 ist in Deutschland inzwischen fast ein Viertel der erwachsenen Bevölkerung adipös (Männer 23,3\%, Frauen 23,9\%). Die häufigste Ursache für
Adipositas ist eine positive Energiebilanz, d.h. es wird mehr Energie über die Nahrung aufgenommen als z.B. durch körperliche Aktivität verbraucht wird. Die Ursache für den Anstieg der Adipositasrate in den Industriekulturen kann allerdings nicht alleine in der Veränderung der Umgebung, z.B. durch ein Überangebot an energiereicher Nahrung und einer bewegungsfeindlichen Umgebung, liegen, da es sehr viele individuelle Unterschiede bezüglich der Gewichtsentwicklung gibt. Daraus ergibt sich, dass die Ursache wahrscheinlich in einer Interaktion von individuellen Verhaltensmustern und der Umgebung zu finden ist. In diesem Kontext wird es spannend, die Rolle des Gehirns zu untersuchen, welches unser Verhalten steuert. Reagiert das Gehirn von Übergewichtigen und Normalgewichtigen unterschiedlich auf Nahrungsreize in der
Umgebung? Wird Hunger anders wahrgenommen? Gibt es elementare Unterschiede in Hirnregionen, die die inhibitorische Verhaltenskontrolle steuern?

Welche genaue Rolle unser Gehirn bei der Entwicklung und Aufrechterhaltung der Adipositas spielt, rückt in den letzten Jahren immer mehr in den Fokus der neurowissenschaftlichen Forschung. In Leipzig forscht ein Verbund aus Neurobiologen, Medizinern, Psychologen und Informatikern als Teil des Integrierten Forschungs- und Behandlungszentrums Adipositas-Erkrankungen unter Beteiligung der Abteilung für Neurologie des Max-Planck-Institutes für Kognitions- und Neurowissenschaften gemeinsam an diesem Thema (www.ifb-adipositas.de, www. sfb-1052.de). Zum Einsatz kommen dabei bildgebende nicht-invasive Methoden, wie die Magnetresonanztomografie (MRT), mit deren Hilfe Hirnstruktur und -funktion beim Menschen untersucht werden können.

\section{Homöostatisches und hedonisches System}

Die zentralnervöse Regulation der Energiebilanz (d.h. die Summe von zugeführter und verbrauchter Energie) ist ein komplexes Zusammenspiel vieler verschiedener Faktoren. Grundlegend können auf der Ebene des Gehirns zwei Hauptanteile beschrieben werden, die jedoch nicht völlig unabhängig voneinander sind: Im homöostatischen System, dessen Regulierung weitestgehend unbewusst über Hormonlevel passiert, senden periphere Botenstoffe, die z.B. in den Verdauungsorganen oder dem Fettgewebe gebildet werden, über den Hypothalamus Informationen an das Gehirn und modulieren dessen Aktivität in verschiedenen Regionen und Netzwerken, um die Nahrungsaufnahme einzuleiten oder zu beenden. Das Hormon Leptin ist zum Beispiel in der Lage, die Aktivität des Belohnungssystems direkt zu modulieren.

Im hedonischen System wird die Nahrungsaufnahme weitestgehend über die Bewertung der sensorischen Eigenschaften oder der Folgen des Verzehrs von Lebensmitteln geregelt. Beide Systeme sind schon sehr alt und nicht nur beim Menschen zu finden. Große Teile des Gehirns sprechen auf Essen, dessen sensorische Eigenschaften (z.B. Aussehen, Geschmack, Geruch; Abbildung 1) oder sogar auf assoziierte Reize an (Kenny 2011). Dabei werden von den verschiedenen Hirnregionen unterschiedliche Funktionen übernommen (Tabelle 1): Die sensorischen Kortizes, wie z.B. der visuelle im Okzipitallappen, der gustatorische im 
Abb. 1: Regionen des menschlichen Gehirns, die als Reaktion auf wohlschmeckendes Essen oder essensassoziierte Reize aktiviert werden (mit freundlicher Genehmigung von Kenny 2009). Der orbitofrontale Kortex und die Amygdala kodieren vermutlich Informationen über den Belohnungswert von Essen. Die Insel verarbeitet Informationen über den Geschmack und Geruch des Essens und seinen hedonischen Wert. Der Nukleus accumbens und das dorsale Striatum, welche beide doaminerge Eingänge aus dem ventralen Tegmentum und der Substantia nigra bekommen, regulieren die motivationalen Eigenschaften des Essens. Der laterale Hypothalamus könnte die Belohnungsantwort auf schmackhaftes Essen modulieren und Nahrungssuche stimulieren. Diese Hirnregionen agieren gemeinsam, um Lernverhalten bezüglich der hedonischen Eigenschaften von Essen zu regulieren, um Aufmerksamkeit und Aufwand auf die Beschaffung belohnender Nahrungsmittel zu richten und um die motivierenden Eigenschaften von Stimuli in der Umgebung, welche mit dem Vorhandensein von belohnendem Essen assoziiert sind, zu regulieren. Aus Gründen der Übersichtlichkeit sind nicht alle Verbindungen zwischen diesen Hirnregionen dargestellt.

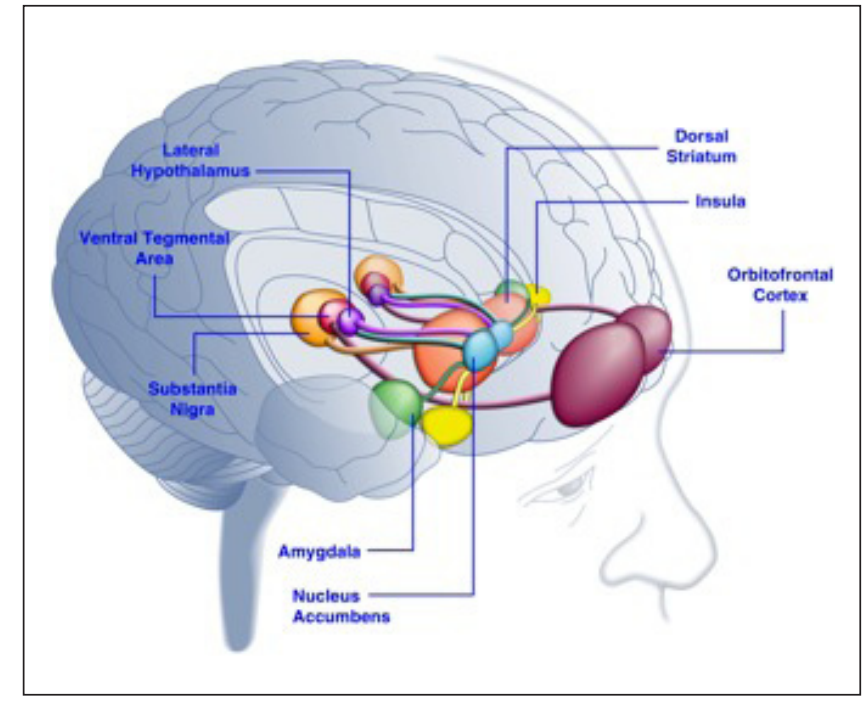

frontalen Operculum oder der olfaktorische im posterioren Orbitofrontalkortex kodieren die modalitätsspezifischen sensorischen Eigenschaften. Der Orbitofrontalkortex übernimmt eine weitgehend modalitätsübergreifende subjektive Bewertung, der anteriore insuläre Kortex integriert interozeptive Signale mit dem subjektiven Wert eines Nahrungsreizes und generiert Motivation.
Das Belohnungssystem in den Basalganglien (Nucleus accumbens, Striatum) signalisiert den subjektiven Belohnungswert von zu erwartetem oder tatsächlich verzehrtem Essen. Die Amygdala fungiert wahrscheinlich als Salienzmarker, welcher einen Reiz mit affektiven Attributen ausstattet, aber auch die Kopplung des Essverhaltens an emotionale Zustände bewerkstelligt.
Essen ist für jeden Organismus essenziell, daher haben sich auf der Ebene des Gehirns komplexe und sehr wirkungsvolle Mechanismen entwickelt, die dafür sorgen, dass energiereiche Nahrung bevorzugt und als angenehm empfunden wird. Der Fall einer übermäßigen Nahrungsaufnahme ist aus evolutionärer Sicht nicht gefährlich und daher anscheinend nicht strikt geregelt.
F

$\overline{\text { FINE SEIENGE TOQLS }}$
FINE SURGICAL INSTRUMENTS FロR RESEARCH ${ }^{\mathrm{TM}}$

\section{Quality surgical instruments as pure as gold.}

Fine Science Tools has been shipping world-renowned surgical and microsurgical instruments globally since 1974 . With offices and dealers throughout the world, FST conveys convenience, expedient and superb customer service with no boundaries.

Visit us at finescience.de to explore our complete product line, and to locate our offices and dealers around the world. 
Tab. 1: Hirnregionen und ihre Rolle bei der Wahrnehmung von Essensreizen und der Regulation des Essverhaltens

\begin{tabular}{|c|c|c|c|c|}
\hline Name & Hirnregion & System & Abkürzung & Funktion im Essenskontext \\
\hline Amygdala & \multirow{2}{*}{$\begin{array}{l}\text { mesiotemporaler } \\
\text { Lappen }\end{array}$} & \multirow{2}{*}{$\begin{array}{l}\text { Limbisches } \\
\text { System }\end{array}$} & & Salienz, Stressantwort \\
\hline Hippocampus & & & & Gedächtnis, Lernen \\
\hline anteriore Insula & Inselkortex & & & $\begin{array}{l}\text { Interozeption, Homöostase, modalitätsunabhängige } \\
\text { Integration sensorischer Signale }\end{array}$ \\
\hline dorsolateraler präfrontaler Kortex & \multirow{4}{*}{ Frontallappen } & & DLPFC & zielgerichtete Verhaltenskontrolle \\
\hline Frontales Operculum & & & & primärer gustatorischer Kortex \\
\hline Orbitofrontaler Kortex & & & OFC & Evaluation von Stimuli, sekundärer gustatorischer Kortex \\
\hline ventromedialer präfrontaler Kortex & & & & $\begin{array}{l}\text { Evaluation von Stimuli, Berechnung eines subjektiven } \\
\text { Wertes }\end{array}$ \\
\hline fusiformer Gyrus & Okzipitallappen & & & visueller Assoziationskortex \\
\hline Hypothalamus & \multirow{2}{*}{ Diencephalon } & & & Integration homöostatischer Information aus dem Körper \\
\hline Thalamus & & & & sensorisches Portal \\
\hline Nucleus accumbens & ventrales Striatum & \multirow{5}{*}{ Basalganglien } & NAcc & Belohnungsprädiktion, Konditionierung \\
\hline Nucleus caudatus & dorsales Striatum & & & Verarbeitung von Feedback \\
\hline Putamen & & & & Vermittlung von automatischem/habituellem Verhalten \\
\hline Substantia nigra & Mittelhirn & & $\mathrm{SN}$ & Kontrolle der Basalganglien, Dopaminversorgung \\
\hline ventrales Tegmentum & & & VTA & $\begin{array}{l}\text { Antwort auf neuartige Stimuli, unerwartete Beloh- } \\
\text { nungen, und mit Belohnung assoziierter Stimuli }\end{array}$ \\
\hline posteriores Cingulum & Parietallappen & & PCC & Episodisches Gedächtnis \\
\hline
\end{tabular}

\section{Funktion von Nahrungsmitteln als natürlicher Verstärker}

Da die Nahrungsaufnahme von essenzieller Bedeutung für den Organismus ist, hat sich ein natürliches Verstärkungssystem entwickelt, welches die Aufnahme physiologisch wertvoller Nahrung fördert. So empfinden wir den Geschmack von zucker- oder fetthaltigen Nahrungsmitteln mit hoher Kaloriendichte als angenehm, das Belohnungssystem des Gehirns sendet entsprechende Signale und die Assoziation zwischen den sensorischen und assoziativen Eigenschaften des Nahrungsmittels und einer positiven Bewertung wird gestärkt. Diese Funktionalität wird wahrscheinlich größtenteils durch den Neurotransmitter Dopamin vermittelt, der sowohl in subkortikalen als auch kortikalen Strukturen eine wichtige Rolle spielt.

Dieses System birgt aber bei einigen Menschen die Gefahr eines unerwünschten Nebeneffektes: Die Verstärkung geht mitunter zu weit und es entwickelt sich suchtähnliches Verhalten. In Analogie zur Suchtforschung wird angenommen, dass Übergewichtige mit der Zeit bei Nahrungsaufnahme eine unzulängliche Belohnungsantwort entwickeln und diese durch vermehrtes Essen kompensieren. Diese Hypothese wurde in mehreren Studien mithilfe der funktionellen Magnetresonanz- tomografie (fMRT) geprüft. Dabei konnte gezeigt werden, dass mit höherem BMI die Antwort auf einen Milchshake im Striatum geringer ausfiel. Dieser Effekt scheint eine Konsequenz und keine Ursache für Adipositas zu sein. Mithilfe der fMRT-Antwort im Striatum gelang eine Vorhersage der Gewichtszunahme nach einem Jahr: Je niedriger die Antwort im dorsalen Striatum war, desto höher war die Gewichtszunahme (Stice, Yokum, Blum und Bohon 2010). In einer Studienpopulation mit erhöhtem Adipositasrisiko durch elterliche Adipositas konnte allerdings kein Zusammenhang zwischen Risiko und Antwort im Belohnungszentrum festgestellt werden.

\section{Neurowissenschaftliche Befunde}

Die MRT liefert verschiedene Maße, mit deren Hilfe Hirnstruktur und -funktion untersucht werden können. T1-gewichtete Bilder bilden die Basis für volumetrische Methoden wie die voxel-basierte Morphometrie (VBM, ein Voxel ist ein dreidimensionales Pixel), bei welcher Volumen und Dichte der Hirnsubstanz für jedes Kompartiment (graue Substanz, weiße Substanz, intrakranielles Volumen) quantifiziert und analysiert werden können.

Mehrere Querschnittsstudien zeigen konsistent geringere Gesamthirnvolumina adipöser Probanden im Vergleich zu normal- oder übergewichtigen Probanden. Zusätzlich war das Volumen der weißen Substanz bei adipösen im Vergleich zu übergewichtigen Probanden verringert. Bei Männern wurde zudem im Gegensatz zu Frauen ein negativer Zusammenhang zwischen BMI und dem Anteil der grauen Substanz am intrakraniellen Gesamtvolumen beobachtet.

Studien, die den Zusammenhang zwischen lokaler Struktur der grauen Substanz und Adipositas mittels VBM untersucht haben, sind bisher zu inkonsistenten Ergebnissen gekommen. Ursächlich dafür könnten einerseits eine erhebliche Varianz im Altersspektrum und andererseits ein variierender Anteil von Männern und Frauen in den untersuchten Populationen sein.

Im Vergleich zu schlanken Teilnehmern hatten adipöse Probanden eine signifikant verringerte Dichte der grauen Substanz in Hirnregionen, die eine Rolle bei der Regulation und Perzeption von Geschmack spielen (Gyrus postcentralis, frontales Operculum), die habituelle Verhaltenskontrolle unterstützen (Striatum, genauer Putamen) und die inhibitorische kognitive Verhaltenskontrolle stützen (dLPFC). Eine andere Studie zeigte bei adipösen Probanden eine generelle Atrophie der Frontallappen, des anterioren Cingulums, des Hippocampus und des Thalamus im Vergleich zu normalgewichtigen Probanden. 
In den letzten Jahren zeigten mehrere Studien, dass das Geschlecht der Probanden ein wichtiger Faktor bei der Untersuchung der Beziehung zwischen Gehirn und Adipositas ist. Eine Studie aus Japan konnte bei Frauen keine verlässlichen Beziehungen zwischen Hirnstruktur und Adipositas nachweisen. Bei Männern hingegen korrelierte das lokale Volumen der grauen Substanz beider Hippocampi und des Precuneus, Regionen die für die Enkodierung und das Abrufen von Erinnerungen wichtig sind, negativ mit dem BMI. Im Gegensatz dazu korrelierte das Volumen der inferioren frontalen Gyri, des Thalamus und der Nuclei Caudata positiv mit dem BMI. Der Altersbereich der Probanden reichte in dieser Studie von 12-81 Jahren. Da Ko-Morbiditäten von Adipositas wie z.B. Diabetes Typ II mit steigendem Alter der Probanden häufiger auftreten, haben wir Adipositas-abhängige Veränderungen der Hirnstruktur an jüngeren Erwachsenen untersucht (Horstmann et al. 2011). Als Korrelationsmaße für den Körperfettgehalt haben wir einerseits den BMI und andererseits den Serumspiegel von Leptin genutzt. Leptin ist ein Hormon, welches proportional zum Körperfettgehalt sezerniert wird und damit ein gutes $\mathrm{Maß}$ für den Fettanteil am Körpergewicht ist. Beide Geschlechter zeigten proportional zu den Übergewichtsmarkern Strukturveränderungen in Hirnregionen, die die individuelle Wertigkeit der Nahrung kodieren (Nucleus accumbens, orbitofrontaler Kortex (OFC)), sowie im Hypothalamus, welcher die Energiehomöostase des Körpers kontrolliert (Abbildung 2, obere Reihe). Beide Befunde könnten ein Essverhalten erklären, bei dem hedonisch motiviertes Essen den homöostatischen Nutzen übersteigt. Die Kausalitätsbeziehung zwischen Hirnbefund und Essverhalten ist aber noch offen. Bei Frauen zeigten sich zusätzlich komplementäre Adipositas-abhängige Veränderungen der Hirnstruktur im dorsalen Striatum (Abbildung 2, untere Reihe) und im dorsolateralen präfrontalen Kortex. Diese Areale vermitteln einerseits habituelles, d.h. gewohnheitsmäßiges, Verhalten (Striatum) und andererseits zielgerichtetes Verhalten (dLPFC).
Die Relevanz dieser Veränderungen in der Hirnstruktur wurde kürzlich durch andere Studien belegt: Eine Studie zeigte, dass reduziertes Volumen im dLPFC mit einer Gewichtszunahme innerhalb eines Jahres assoziiert war, eine andere konnte belegen, dass Menschen, die gerade eine Diät einhalten, eine erhöhte Aktivierung in dieser Hirnregion haben, wenn sie Entscheidungen über den Verzehr von Nahrungsmitteln fällen (Hare, Camerer und Rangel 2009). Dies könnte bedeuten, dass ein geringeres Volumen von Hirnarealen, die an der inhibitorischen Verhaltenskontrolle beteiligt sind, mit einer perspektivischen Gewichtszunahme in Verbindung steht.

Die diffusionsgewichtete Bildgebung (engl. Diffusion tensor imaging, DTI) misst die Diffusivität von Wassermolekülen im Gewebe und hat sich als äußerst nützlich dabei erwiesen, die Architektur und Integrität der weißen Substanz des Gehirns zu untersuchen. Da die Zellmembranen und Myelinschichten der Neurone natürliche Barrieren darstellen, ist die Diffusivität orthogonal zur Faserrichtung (radiale Dif-
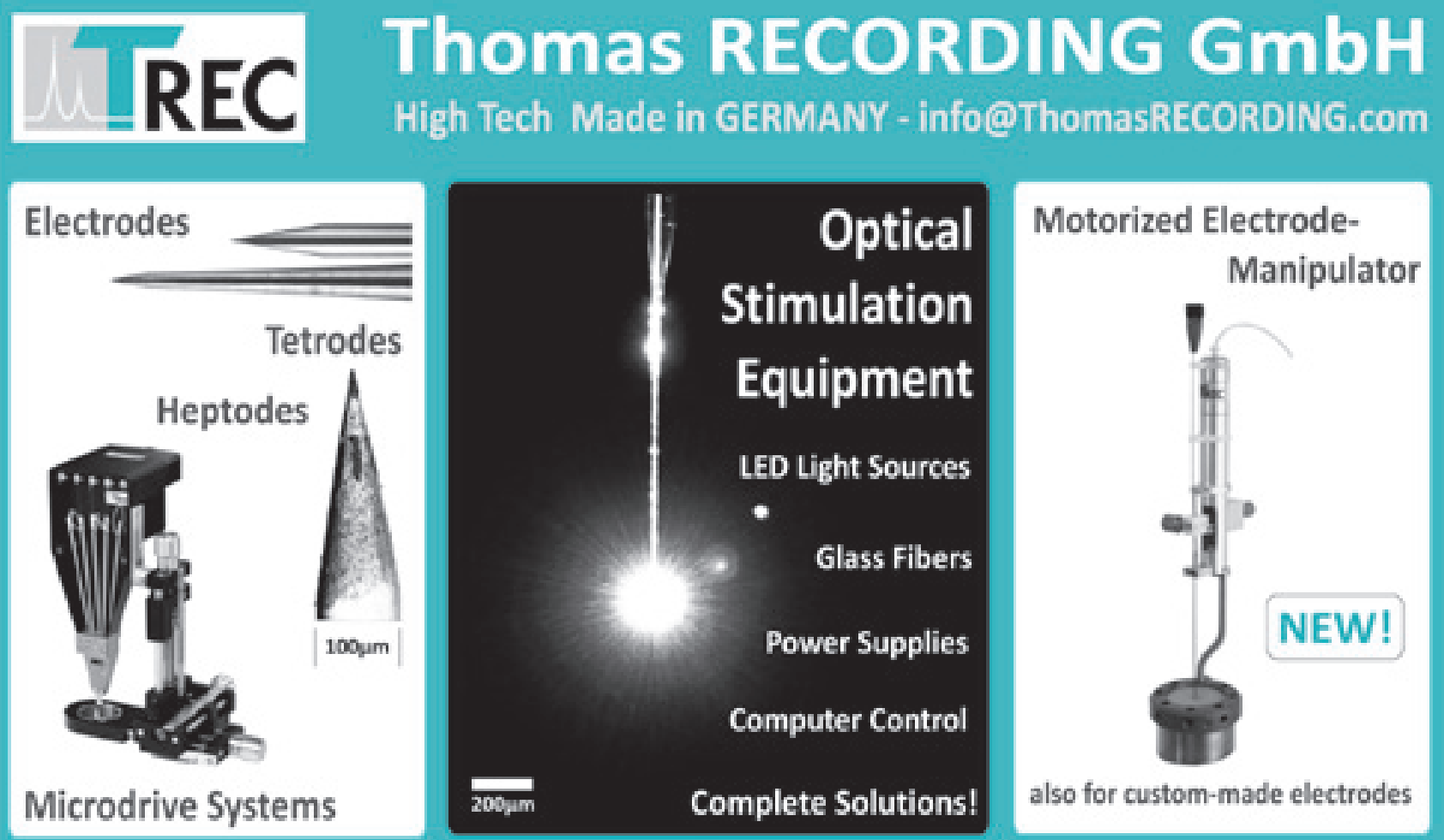

For more than 20 years complete Neuro-Laboratory Equipment available from: 


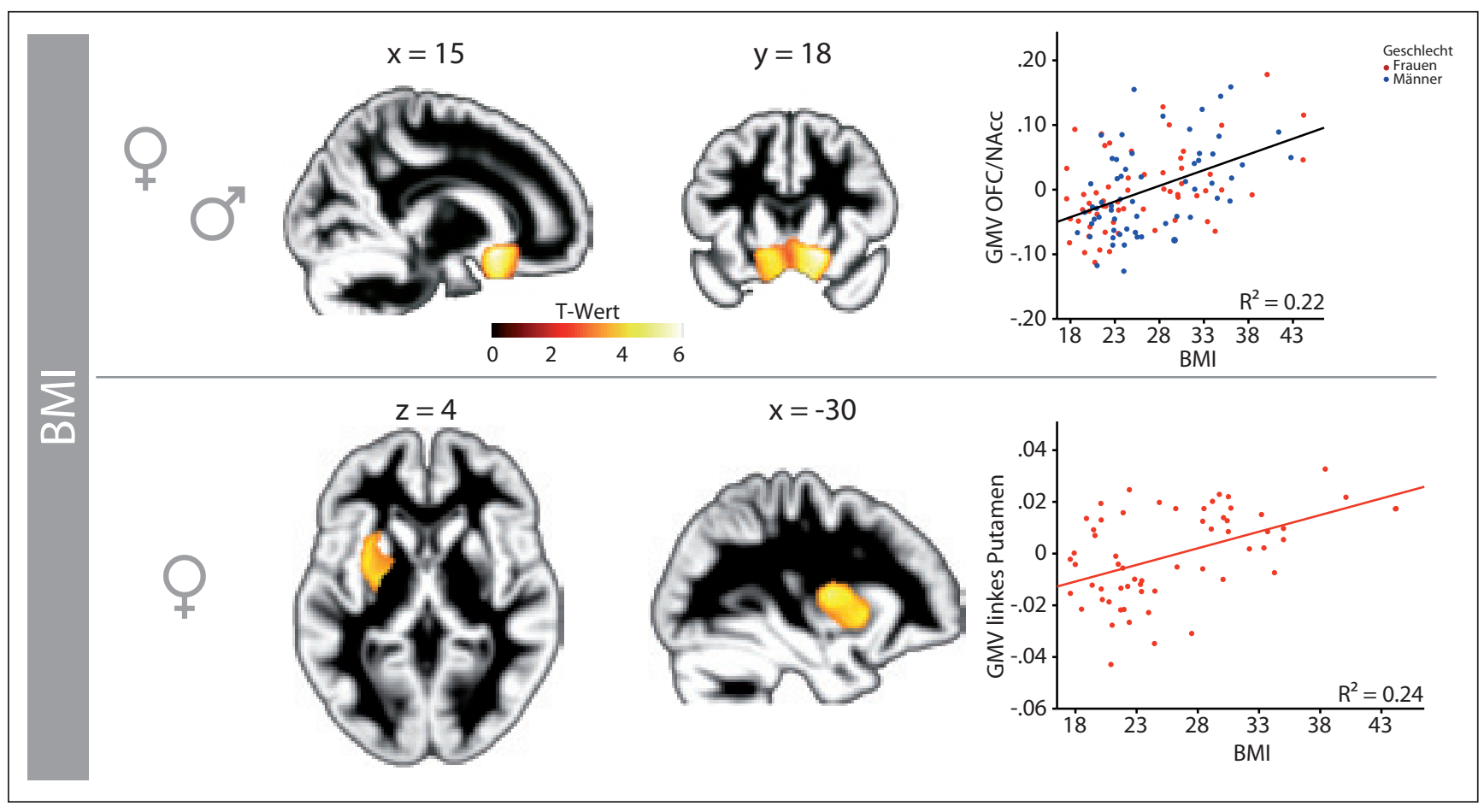

Abb. 2: Adipositas ist mit geschlechtsabhängigen Veränderungen der Struktur in Hirnarealen assoziiert, welche ein Rolle bei der Belohnungsverarbeitung und der kognitiven sowie homöostatischen Kontrolle spielen. Das Volumen des posterioren medialen Orbitofrontalen Kortex (OFC), des Nucleus accumbens (NAcc) und des Hypothalamus steigt signifikant mit steigendem BMI (obere Reihe, warme Tönung; Streudiagramm) in beiden Geschlechtern. Für Frauen konnte zusätzlich eine positive Assoziation zwischen dem Volumen der grauen Substanz und dem BMI im Putamen beobachtet werden (untere Reihe: warme Tönung; Streudiagramm). Quelle: Horstmann et al. 2011

fusivität) geringer als diejenige entlang der Trakte (axiale Diffusivität). Die Fraktionale Anisotropie (FA) ist ein Index, der das Ungleichgewicht zwischen diesen unterschiedlichen Diffusivitäten quantifiziert und ist ein sensitiver Marker für Veränderungen in der Mikrostruktur der weißen Substanz.

In zwei unabhängigen Kohorten wurde ein ähnlicher negativer Zusammenhang zwischen dem BMI und den Diffusionsparametern im Corpus Callosum und dort vor allem im Bereich des Spleniums beobachtet (Mueller et al. 2011; Stanek et al. 2011), was für einen relativ stabilen Effekt spricht. Auch hier spielt das Geschlecht der betroffenen Personen wieder eine Rolle: Bei Frauen war der Effekt viel größer als bei den Männern in der Studie. Zusätzlich scheint es eine Interaktion zwischen den Effekten von Adipositas und Alterungsprozessen auf die Mikrostruktur der weißen Substanz zu geben: Alterungsprozesse verstärken die beobachteten Effekte zusätzlich.

Eine mögliche Verbindung zwischen der Integrität der Hirnsubstanz und Adipositastypischen Stoffwechselstörungen wurde kürzlich vorgeschlagen: Jugendliche mit metabolischem Syndrom hatten, abhängig von der Stärke des Syndroms, kleinere Hippocampi, einen verringerten Anteil des Gehirns am gesamten intrakraniellen Volumen und eine Reduktion der mikrostrukturellen Integrität in Hauptfasertrakten der weißen Substanz. Voxel-weise Korrelationen zwischen dem Cholesterolprofil der Probanden und der FA ihres Gehirns ergab eine negative Abhängigkeit in beiden Frontallappen. Dies könnte bedeuten, dass auch die Befunde der anderen Studien eventuell auf ein abnormes Cholesterolprofil zurückzuführen sind. Die Konzentration an Fibrinogen, einem Entzündungsmarker, ist normalerweise bei übergewichtigen und adipösen im Vergleich zu schlanken Probanden mit gleicher Alters- und Geschlechtsverteilung erhöht. Korreliert man diesen mit dem Volumen der grauen Substanz, so wird ein negativer Zusammenhang zwischen Fibrinogen und dem Orbitofrontalen Kortex deutlich. In der gleichen Gruppe von Probanden konnte eine positive Assoziation zwischen der Konzentration an Fibrinogen und der Diffusivität in der grauen Substanz von Amygdala und des Parietalkortex gezeigt werden. Diese Daten legen die Interpretation nahe, dass ein Teil der hirnanatomischen Befunde auf Adipositas-assoziierte Entzündung oder metabolische Auffälligkeiten zurückzuführen sind.
Die funktionelle Konnektivität zwischen verschiedenen Teilen des Gehirns wird anhand der Beziehung ihrer Signaländerungen über die Zeit gemessen. Eine Gruppe von Regionen, deren Signalzeitverläufe über eine bestimmte Zeit sehr ähnlich sind, kann als funktionelles Netzwerk interpretiert werden. Ohne externe Stimulation wird es als Resting-State-Netzwerk (RSN) bezeichnet. Die funktionelle Konnektivität spiegelt dabei wahrscheinlich die Koordination der Aktivität unterschiedlicher neuronaler Subpopulationen wider, die entsteht, wenn eine komplexe Berechnung innerhalb eines Netzwerkes von Hirnregionen geleistet wird. Auch in diesem Maß zeigen die Gehirne von Schlanken und Adipösen Unterschiede.

Hirnregionen, welche mit der Verarbeitung von Essen und Belohnungsreizen im Allgemeinen betraut sind, waren in der Stärke ihrer funktionellen Konnektivität sensitiv gegenüber dem peripheren Hormon Insulin: Im orbitofrontalen Kortex und dem Striatum war die funktionelle Konnektivität negativ mit der Insulinsensitivität assoziiert. Wurde Insulin intranasal gegeben, so modulierte es die Konnektivität zwischen Hypothalamus und orbitofrontalem Kortex (Kullmann et 


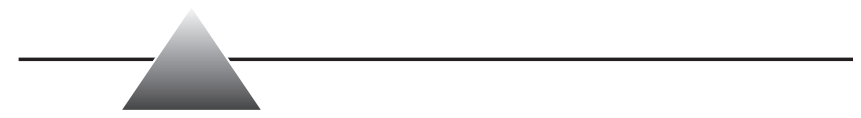

al. 2012). Dies könnte darauf schließen lassen, dass hormonelle Signale die Nahrungsaufnahme reduzieren, indem sie direkt die intrinsische Aktivität des Gehirns modifizieren.

Im Vergleich zu normalgewichtigen Frauen zeigten adipöse Frauen eine verringerte Modulation der Aktivität im orbitofrontalen Kortex und im Belohnungssystem durch die Amygdala während sie Essensbilder betrachteten. Zusätzlich wurde die Aktivität im Belohnungssystem stärker durch den orbitofrontalen Kortex moduliert. Der verringerte Einfluss aus der Amygdala könnte dafür sorgen, dass die affektiven Aspekte der Nahrungsmittel suboptimal moduliert werden. Die gesteigerte Konnektivität zwischen orbitofrontalem Kortex und dem Belohnungssystem spricht eventuell für eine höhere motivationale Salienz von Nahrungsreizen. Adipositas-abhängige Veränderungen in den Basalganglien beziehen sich nicht ausschließlich auf die Hirnstruktur, sondern auch auf die funktionelle Konnektivität während der Bewertung von Nahrungsmitteln. Adipöse Probanden zeigten stärkere funktionelle Verbindungen sowohl zwischen dem Putamen und dem Salienznetzwerk als auch zwischen dem Nucleus caudatus einerseits und Amygdala und Insula andererseits.

Zusammengenommen könnten die zwischen Normal- und Übergewichtigen beschriebenen Unterschiede in Struktur und Interaktion von Hirnregionen, die an der Verarbeitung von Affekten, der Berechnung des motivationalen Wertes von Reizen, an der Gedächtnisbildung und an inhibitorischer Verhaltenskontrolle beteiligt sind, zum Überkonsum bestimmter Nahrungsmittel beitragen. Darüber hinaus scheinen mit Adipositas assoziierte metabolische Veränderungen ungünstige Veränderungen der Hirnsubstanz zu fördern.

Da die meisten der beschriebenen Studien Querschnittsstudien sind, ist eine wichtige offene Frage die nach Ursache und Effekt. Momentan laufen an mehreren Orten Längsschnittstudien an der normalen Bevölkerung oder an speziellen Patientengruppen (z.B. Patienten, die sich einer operativen Magenverkleinerung unterziehen), um Aufschlüsse darüber zu erhalten, welche Veränderungen im Laufe der Entwicklung einer Adipositas entstehen bzw. welche durch einen massiven Gewichtsverlust wieder rückgängig gemacht werden können.

Der Großteil der Studien, die die Antwort des Gehirns auf z.B. Nahrungsreize untersucht hat, ist bisher an Frauen durchgeführt worden. Verallgemeinerbare Schlussfolgerungen zum Zusammenhang zwischen Adipositas und der Hirnantwort sind daher bisher schwierig.

\section{Genetische Einflüsse auf Adipositas und Essverhalten}

Die sogenannte „Hypothese der wirtschaftlichen Gene“ postuliert, dass unser heutiger Genpool durch positive Selektion im Hinblick auf die Fähigkeit, Energiereserven für Zeiten schlechter Versorgung anzulegen, entstanden ist. Menschen, die besonders gut Energie speichern konnten, hatten demnach über lange Zeit hinweg bessere Überlebenschancen und waren damit auch erfolgreicher darin, ihre Gene weiterzugeben. In der heutigen Zeit gibt es in vielen Teilen der Welt ein Überangebot an energiereicher Nahrung. Hinzu kommt, dass im Allgemeinen weniger Energie im täglichen Leben verbraucht wird. Dies könnte demnach in der heutigen Zeit ganz automatisch zu Adipositas führen. Im letzten halben Jahrhundert haben sich Gesellschaft und Umweltbedingungen in vielen Gegenden der Welt sehr schnell geändert; schneller jedenfalls, als sich unser Genpool daran anpassen könnte.

Nach heutigem Stand der Forschung wird das Gewicht zu 40 bis $70 \%$ an die Nachkommen weitergegeben. Dabei spielen neben

\section{Electronic Instruments for the Life Sciences}

\section{made to measure}

\section{NEW Low-Price Instrument Series}

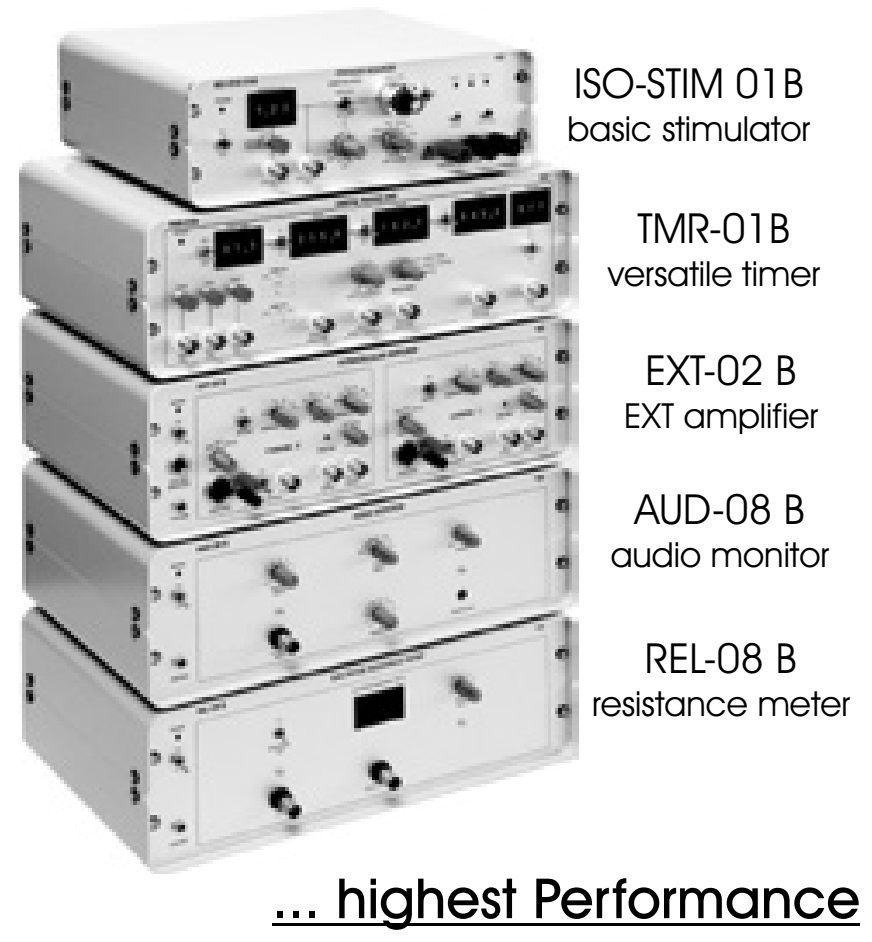

\section{npi provides complete rigs for electrophysiology}

\author{
npi is distributing:
}

- ALA Scientific perfusion systems and accessories

- Burleigh micromanipulators and mounts

- Campden vibrating microtomes

- DataWave data acquisition systems

- Dragonfly commutators with up to 64 lines

- Lumen Dynamics X-Cite fluorescence illumination

- Molecular Devices amplifiers and data acquisition

- NeuroNexus acute and chronic electrodes

- Scientifica micromanipulators, mounts, Slicescope, two-photon Slicescope

- Sensapex piezo driven micromanipulator

- TMC vibration isolation tables and Faraday cages

\section{npi electronic $\mathrm{GmbH}$ \\ Bauhofring 16, D-71732 Tamm}

Phone + 49 (0)7141-97302-30; Fax: + 49 (0)7141-97302-40

support@npielectronic.com; http://www.npielectronic.com 


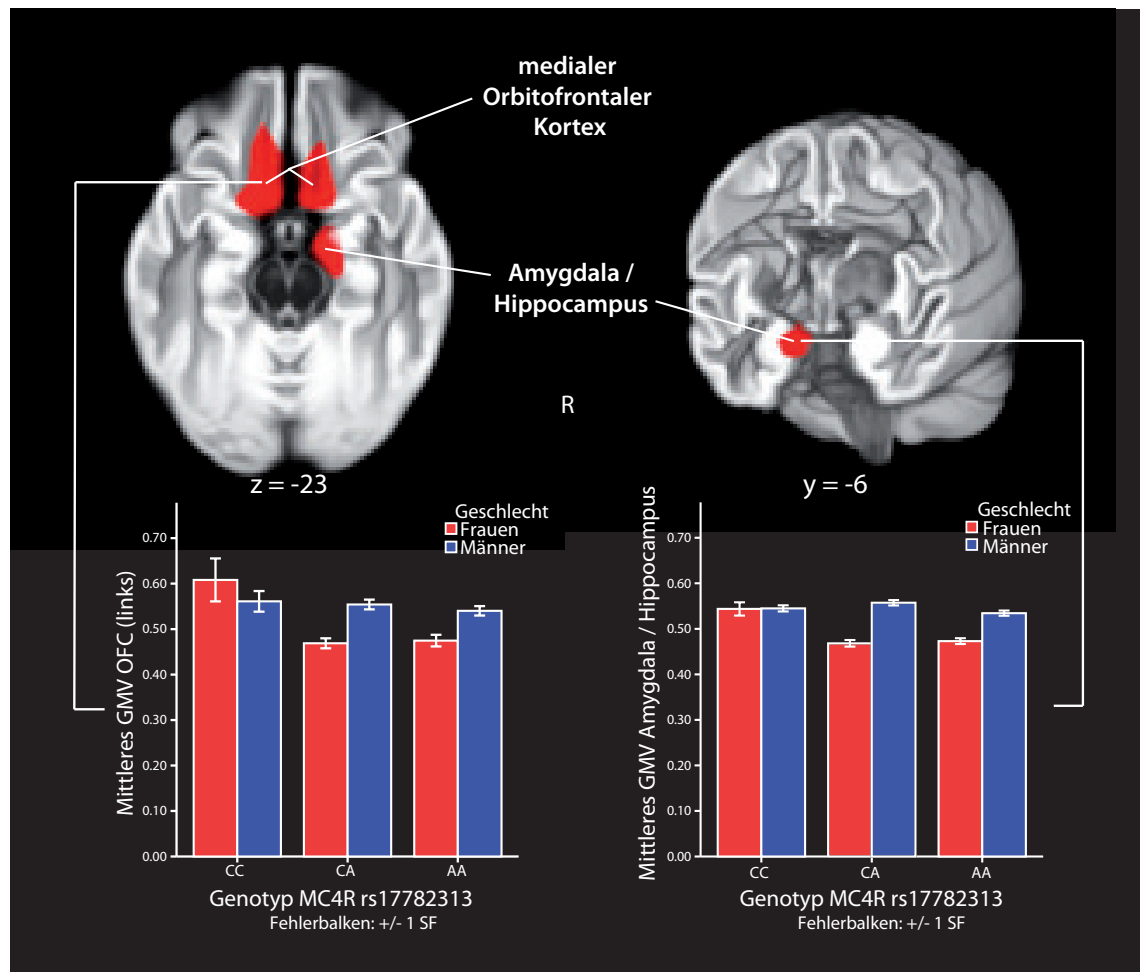

Abb. 3: Geschlechtsspezifische Assoziation zwischen genetischer Variation in der Nähe des MC4R-Gens und dem Volumen der grauen Substanz. Genetische Variation in der Nähe des MC4R-Gens ist mit Adipositas assoziiert. Geschlechtsspezifischer Effekt des Genotypen in der rechten Amygdala/Hippocampus und im bilateralen medialen Orbitofrontalen Kortex, korrigiert für Alter und BMI der Probanden. Weibliche homozygote Träger des Adipositas-Risikoallels (CC) haben höhere Werte als heterozygote Probandinnen (AC) oder Nichtträger (AA). Für Männer wurden keine signifikanten Einflüsse festgestellt. Quelle: Horstmann et al. 2013

genetischen Ursachen auch kulturelle und soziale Faktoren eine große Rolle, d.h. es finden stets Wechselwirkungen zwischen Genen und Umweltbedingungen statt. In den letzten zehn Jahren hat die Forschung zu den genetischen Ursachen der Adipositas durch technische Neuerungen erhebliche Fortschritte gemacht. Man kennt heute neun Gene, in welchen Mutationen zu übermäßigem Essen und starker Adipositas führen. Obwohl jedes dieser Gene einen starken Einfluss auf das Körpergewicht hat, kommen sie sehr selten vor (zusammen genommen nur in ungefähr in 5 bis $10 \%$ der stark Übergewichtigen) und können daher nur einen kleinen Teil des Anteils adipöser Menschen erklären.

Dem gegenüber stehen viele häufige genetische Varianten, die jede für sich nur einen kleinen Einfluss auf das Körpergewicht haben. Meist handelt es sich dabei um sogenannte ,single nucleotide polymorphisms" (SNPs), bei denen es sich um Variationen einzelner Basenpaare in einem DNA-Strang handelt. Diese werden in genom-weiten Assoziationsstudien
(GWAS) identifiziert, indem der Einfluss jeder einzelnen Variante auf Phänotypen wie z.B. Adipositas bestimmt wird. Man geht davon aus, dass viele verschiedene SNPs gemeinsam bestimmen, wie ein Merkmal ausgeprägt ist, wie es zum Beispiel für die Körpergröße der Fall ist. Nimmt man den Einfluss aller bis heute mit Übergewicht in Verbindung gebrachten SNPs zusammen, so erklären sie allerdings nur ungefähr 2 bis 4\% der Varianz im Körpergewicht.

Interessant ist allerdings, dass fast alle dieser SNPs ihren Einfluss über die Wahrnehmung von Hunger und Sättigung bzw. die Nahrungsaufnahme ausüben und damit einen direkten Bezug zum Gehirn haben; manche beeinflussen sogar die Hirnentwicklung (Speliotes et al. 2010; Willer et al. 2009). Dies legt den Schluss nahe, dass vor allem das Essverhalten genetisch bestimmt wird (d.h. was, wann und wie viel gegessen wird) und weniger die Verstoffwechslung der aufgenommenen Kalorien. Trotz des geringen Einflusses einzelner SNPs auf das individuelle Ge- wicht konnten wir in eigenen Studien konsistente Unterschiede im Essverhalten und parallel sogar Veränderungen des Gehirns abhängig vom Genotyp zeigen (Horstmann et al. 2013).

Trägerinnen einer mit Adipositas assoziierten Veränderung nahe eines Melanocortinrezeptors (MC4R) zeigten gegenüber Trägerinnen des Wildtyps ein deutlich enthemmtes Essverhalten, welches mit strukturellen Veränderungen des Gehirns in der Amygdala, dem orbitofrontalen Kortex und dem dorsolateralen Präfrontalkortex (dLPFC) einhergingen (Abbildung 3). $\mathrm{Ob}$ diese Veränderungen ursächlich für den genetischen Einfluss auf das Gewicht sind, bleibt jedoch noch zu klären.

Für einige dieser SNPs ist jedoch bereits belegt, dass deren Einfluss auf das Körpergewicht durch sportliche Aktivität minimiert werden kann. Dies spricht für eine genetisch bedingte Prädisposition, die nicht in jedem Fall zu Übergewicht führt, sondern deren Ausprägung erheblich durch Lebensstil und Umweltbedingungen beeinflusst werden kann. So führen z.B. auch soziale Faktoren wie die Einkommensungleichheit innerhalb einer Gesellschaft zu einer höheren Adipositasrate.

Die Gene bestimmen sicherlich zu einem Großteil, ob sich ein einzelnes Individuum eher am unteren oder am oberen Rand der Gewichtsverteilung befindet. Adipositas wird allerdings in den seltensten Fällen ausschließlich vererbt. Die allgemeine Verschiebung der Gewichtsverteilung hin zu einem höheren Durchschnittsgewicht ist damit in der Hauptsache wahrscheinlich ein Phänomen unserer modernen Gesellschaft.

\section{Zusammenfassung}

Adipositas scheint in den meisten Fällen durch ein verändertes Essverhalten hervorgerufen zu werden. Evolutionär hat sich kein Mechanismus entwickelt, der zuverlässig vor einer zu hohen Nahrungsaufnahme schützt. Bisher konnten viele Studien zeigen, dass es komplexe Unterschiede in der Struktur und intrinsischen Informationsverarbeitung zwischen den Gehirnen von normalgewichtigen und adipösen Menschen gibt. Ob diese Unterschiede Ursache oder Konsequenz der Adipositas sind, bleibt in den meisten Fällen noch zu klären. Genetische Variation, die mit Adipositas in Zusammenhang gebracht wurde, wirkt sich zum Teil auch auf Aufbau und Funktion des Gehirns aus. Offene Punkte sind die Spezifität der beobachteten Unterschiede für den Essenskontext und die eventuelle Umkehrbarkeit als Therapieansatz. 


\section{Danksagung}

Diese Arbeit wurde vom IFB Adipositas Erkrankungen, Bundesministerium für Bildung und Forschung (BMBF, www.bmbf. de), Deutschland, FKZ: 01E01001 und der Deutschen Forschungsgemeinschaft (DFG, www.dfg.de) im Rahmen des Sonderforschungsbereiches 1052 „Mechanismen der Adipositas", Projekte A1 und A5, gefördert.

\section{Literatur}

Hare, T. a, Camerer, C. F. und Rangel, A. (2009): Self-control in decision-making involves modulation of the vmPFC valuation system. Science (New York, N.Y.) 324 (5927): 646-8. doi:10.1126/science.1168450

Horstmann, A., Busse, F., Mathar, D., Mueller, K., Lepsien, J., Schloegl, H. und Pleger, B. (2011): Obesity-related differences between women and men in brain structure and goaldirected behavior. Frontiers in Human Neuroscience 5. doi:10.3389/fnhum.2011.00058

Horstmann, A., Kovacs, P., Kabisch, S., Boettcher, Y., Schloegl, H., Tönjes, A. und Villringer, A. (2013): Common Genetic Variation near MC4R Has a Sex-Specific Impact on Human Brain Structure and Eating Behavior. (F. J. Esteban, Ed.) PLoS ONE 8 (9): e74362. doi:10.1371/journal.pone.0074362

Kenny, P. J. (2011): Reward mechanisms in obesity: new insights and future directions. Neuron 69 (4): 664-79. doi:10.1016/j.neuron.2011.02.016

Kullmann, S., Heni, M., Veit, R., Ketterer, C., Schick, F., Häring, H.-U. und Preissl, H. (2012): The obese brain: association of body mass index and insulin sensitivity with resting state network functional connectivity. Human brain mapping 33 (5): 1052-61. doi:10.1002/ hbm. 21268

Mueller, K., Anwander, A., Möller, H. E., Horstmann, A., Lepsien, J., Busse, F. und Pleger, B. (2011): Sex-dependent influences of obesity on cerebral white matter investigated by diffusion-tensor imaging. PLOS ONE (February): 1-30.

Speliotes, E. K., Willer, C. J., Berndt, S. I., Monda, K. L., Thorleifsson, G., Jackson, A. U. und Mägi, R. (2010): Association analyses of 249,796 individuals reveal 18 new loci associated with body mass index. Nature genetics 42 (11): 937-48. doi:10.1038/ng.686
Stanek, K. M., Grieve, S. M., Brickman, A. M., Korgaonkar, M. S., Paul, R. H., Cohen, R. A. und Gunstad, J. J. (2011): Obesity is associated with reduced white matter integrity in otherwise healthy adults. Obesity (Silver Spring, Md.) 19 (3): 500-4. doi:10.1038/oby.2010.312 Stice, E., Yokum, S., Blum, K. und Bohon, C. (2010): Weight Gain Is Associated with Reduced Striatal Response to Palatable Food. Journal of Neuroscience 30 (39): 13105-13109. doi:10.1523/JNEUROSCI.2105-10.2010

Willer, C. J., Speliotes, E. K., Loos, R. J. F., Li, S., Lindgren, C. M., Heid, I. M. und Lamina, C. (2009): Six new loci associated with body mass index highlight a neuronal influence on body weight regulation. Nature genetics 41 (1): 25-34. doi:10.1038/ng.287

\section{Kurzbiografien}

Arno Villringer (geb. 1958 in Schopfheim), studierte Medizin in Freiburg. Nach einem einjährigen Aufenthalt an der Harvard Universität war er von 1986 bis 1993 als Assistenzarzt an der Universität München tätig. Von 1996 bis 2003 war er leitender Oberarzt und stellvertretender

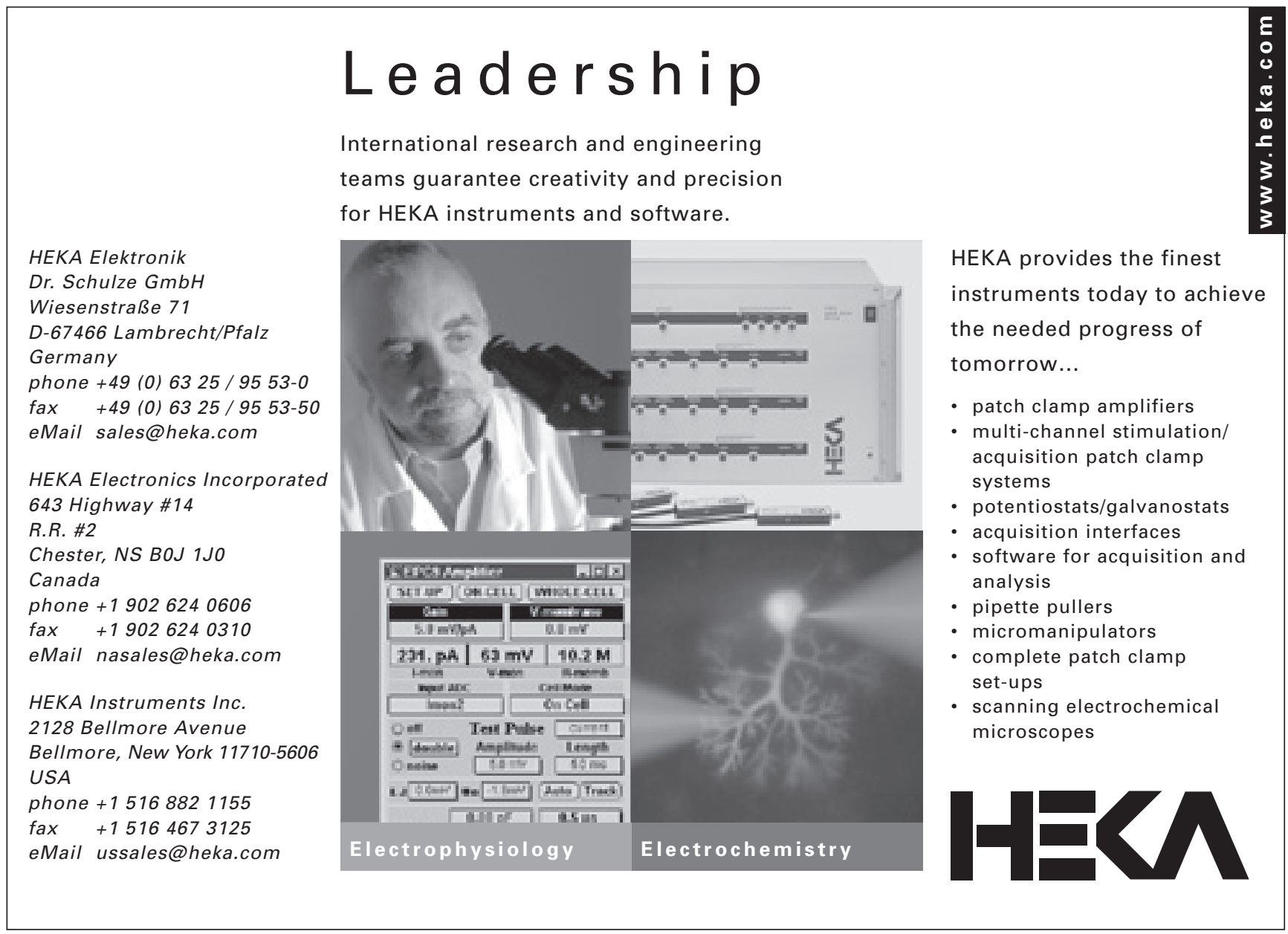


Klinikdirektor an der Neurologischen Klinik der Charité, von 2004 bis 2007 Klinikdirektor am Campus Benjamin Franklin der Charité. Seit 2007 ist er Direktor am MPI für Kognitions- und Neurowissenschaften sowie Klinikdirektor der Klinik für Kognitive Neurologie am Uniklinikum Leipzig. Prof. Villringer leitet seit 1999 das bundesweite Kompetenznetz Schlaganfall und hat 2006 im Rahmen der Exzellenzinitiative die Berlin School of Mind and Brain gegründet, deren Sprecher er seither ist. Sein Forschungsschwerpunkt sind der Schlaganfall und seine Risikofaktoren.

Annette Horstmann (geb. 1977 in Hamburg), studierte Biologie an der RuhrUniversität in Bochum. Nach einem einjährigen Forschungsaufenthalt am Max-Planck-Institut für Kognitions- und Neurowissenschaften in Leipzig (Arbeitsgruppe „Attention \& Awareness“, Prof. John-Dylan Haynes) und der Promotion im Rahmen der „Internationalen Graduiertenschule Biowissenschaften“ in Bochum war sie von 2008 bis 2010 als PostDoc in der Abteilung für Neurologie desselben Institutes tätig. Von 2010 bis 2013 arbeitete sie als wissenschaftliche Mitarbeiterin in einem Kooperationsprojekt zwischen dem MPI und dem IFB Adipositas-Erkrankungen, Universitätsmedizin Leipzig. Dr. Horstmann leitet seit 2013 die eigenständige Nachwuchsforschungsgruppe „Entscheidungsverhalten bei Adipositas: Neurobiologie, Verhalten und Plastizität" im Rahmen des IFB Adipositas-Erkrankungen in Kooperation mit dem MPI für Kognitions- und Neurowissenschaften. Ihr Forschungsschwerpunkt sind die neurobiologischen Grundlagen der Adipositas beim Menschen.

\section{Korrespondenzadressen}

\section{Dr. Annette Horstmann}

Max-Planck-Institut für Kognitions-

und Neurowissenschaften

Kognitive Neurologie

Stephanstr. $1 \mathrm{~A}$

04103 Leipzig

Tel.: $\quad+4934199402258$

E-Mail: horstmann@cbs.mpg.de

\author{
Prof. Dr. Arno Villringer \\ Max-Planck-Institut für Kognitions- \\ und Neurowissenschaften \\ Kognitive Neurologie \\ Stephanstr. $1 \mathrm{~A}$ \\ 04103 Leipzig \\ Tel.: $\quad+4934199402220$ \\ E-Mail: villringer@cbs.mpg.de
}

\title{
Lieferung auf Abruf: Exosomen als "Care“-Pakete von Gliazellen für gestresste Neurone
}

\author{
Eva-Maria Krämer-Albers und Carsten Frühbeis
}

Zusammenfassung

Die Kommunikation zwischen Zellen ist eine grundlegende Voraussetzung für reibungslose Abläufe im Nervensystem. Gliazellen besitzen dabei eine Vielzahl von Aufgaben, die in enger Abstimmung mit Neuronen wahrgenommen werden. Forschungen der letzten Jahre zeigen, dass Zellkommunikation auch über den Austausch von extrazellulären Vesikeln stattfindet, die ebenfalls von Gliazellen und Neuronen sezerniert werden. Zu den extrazellulären Vesikeln gehören Exosomen und Mikrovesikel, welche Proteine und Ribonukleinsäuren zu Zielzellen transportieren. Nach erfolgtem Transfer können diese Komponenten dann den Phänotyp der Zielzelle verändern. In diesem Artikel diskutieren wir Eigenschaften und Funktionen von extrazellulären Vesikeln im Allgemeinen und speziell im zentralen Nervensystem. Dort geben myelinisierende Oligodendrozyten in Antwort auf Neurotransmittersignale Exosomen ab, die von Neuronen aufgenommen werden und neuroprotektive Eigenschaften besitzen.

\section{Abstract}

Delivery on call: Exosomes as „Care packages“ from glia cells for stressed neurons. Communication between cells is a basic requirement for proper nervous system function. Glia cells execute versatile functions, which operate in close coordination with neurons. Recent research revealed that cell communication is mediated by the exchange of extracellular vesicles, which are also secreted by glia cells and neurons. Extracellular vesicles comprise exosomes and microvesicles, which deliver proteins and ribonucleic acids to target cells. As a result of transfer, the vesicle cargo components can modulate the phenotype of recipient cells. Here, we discuss the characteristics and functions of extracellular vesicles in general and in particular in the central nervous system. There, myelinating oligodendrocytes release exosomes in response to neurotransmitter signals, which are internalized by neurons and exhibit neuroprotective functions.

Keywords: oligodendrocytes; extracellular vesicles; exosomes; cell-cell communication; axon-glia interaction

\section{Einleitung}

Gliazellen partizipieren aktiv an den verschiedensten Prozessen im Nervensystem. Die Vorstellung vom ursprünglich durch Rudolf Virchow terminierten „Leim“ des Nervengewebes ist längst überholt und die Liste der Fähigkeiten von Gliazellen erweitert sich kontinuierlich. Mikroglia, Astrozyten und Oligodendrozyten sind sowohl für die Entwicklung als auch für die alltäglichen Funktionen des Gehirns von enormer Bedeutung: Sie steuern das Wachstum von Axonen, gewährleisten die Nährstoffversorgung von Neuronen, modulieren Signalverarbeitungsprozesse, sorgen für elektrische Isolation (Myelinbil- dung) und kontrollieren Immunprozesse. Die Koordination dieser Abläufe hängt in besonderem Maße von der Kommunikation zwischen Gliazellen und Neuronen ab. Nach der klassischen Sicht findet Zellkommunikation entweder durch direkten Zellkontakt oder die parakrine Wirkung löslicher Mediatoren statt. Allerdings hat man im Laufe des letzten Jahrzehnts festgestellt, dass auch ganze Vesikel zwischen Zellen ausgetauscht werden, die eine Kollektion von Biomolekülen, gleichsam als Paket, zu Zielzellen transportieren können. Die ,angelieferten“ Moleküle können dann direkt in den Zielzellen agieren (Thery 2011). Aktuelle Forschungen zeigen, dass auch die Zellen des Nervensystems Vesikel 